Organo- and

Biocatalysis

\title{
Chiral Ammonium Betaines as Bifunctional Organocatalysts
}

Key words

ammonium betaines

bifunctional

catalysts

Mannich reaction

betaine catalysis

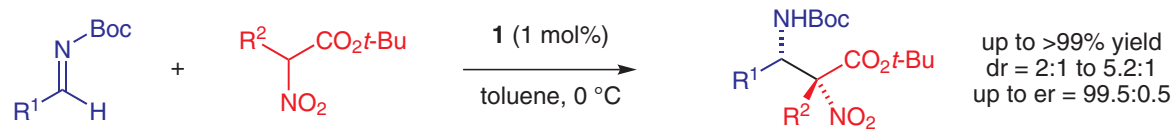

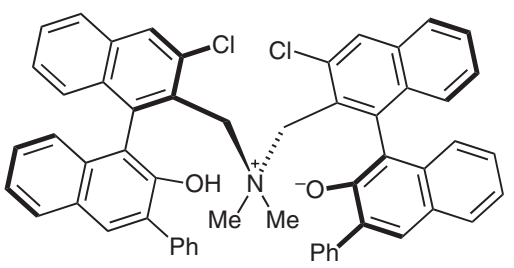

1

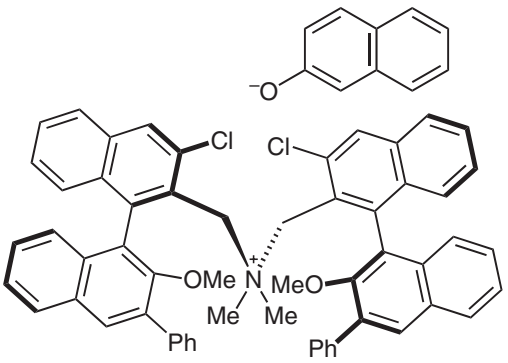

2

Only the betaine catalyst is selective:

Betaine

Structured ion-pair
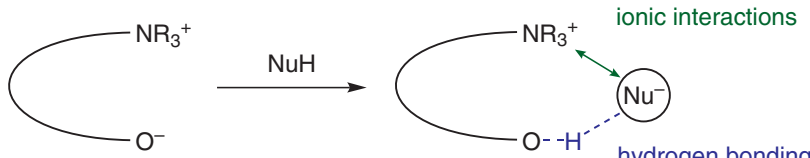

Significance: The authors established a new ammonium betaine catalyst in asymmetric Mannichtype reactions. The reaction of $\alpha$-nitrocarboxylates with $N$-Boc imines proceeded in the presence of catalyst $\mathbf{1}$ in excellent yields and enantioselectivities, albeit diastereoselectivities of the products were only moderate, in favor of the syn isomer. Although the activity was comparable to betaine $\mathbf{1}$, almost no stereoselectivity was observed when catalyst 2 was used. This points out the crucial role of the betaine structure, since it is able to form a structured ion pair in the reaction course.
Comment: In the present work a new class of organocatalysts is introduced. Although the reported reaction is well established in organocatalysis (e.g., A. Singh, J. N. Johnston J. Am. Chem. Soc. 2008, 130, 5866), this work demonstrates the power of new bifunctional ammonium betaines like $\mathbf{1}$ as enantioselective organic base catalysts. Especially the high enantioselectivities at low catalyst loadings (1 mol\%) are remarkable. The importance of the zwitterionic nature of the catalysts was shown nicely. Reports on new organic betaine catalysts and further applications can be anticipated.

SYNFACTS Contributors: Benjamin List, Steffen Müller 